\title{
OPTIMAL ERROR ESTIMATES FOR THE STOKES AND NAVIER-STOKES EQUATIONS WITH SLIP-BOUNDARY CONDITION
}

\author{
EBERHARD BÄNSCH${ }^{1}$ AND KLAUS DECKELNICK ${ }^{2}$
}

\begin{abstract}
We consider a finite element discretization by the Taylor-Hood element for the stationary Stokes and Navier-Stokes equations with slip boundary condition. The slip boundary condition is enforced pointwise for nodal values of the velocity in boundary nodes. We prove optimal error estimates in the $H^{1}$ and $L^{2}$ norms for the velocity and pressure respectively.
\end{abstract}

AMS Subject Classification. 35Q30, 65N15, 65N30, 76D05, 76D07.

Received: February 9, 1998. Revised: November 27, 1998.

\section{INTRODUCTION}

We consider the stationary Navier-Stokes equations: given a bounded domain $\Omega \subseteq \mathbb{R}^{d}, d=2$ or $d=3$, find a velocity field $u$, a pressure $p$ such that

$$
-\frac{1}{\operatorname{Re}} \Delta u+(u \cdot \nabla) u+\nabla p=f \quad \text { in } \Omega
$$

as well as its linear counterpart, the Stokes equations:

$$
\begin{aligned}
-\frac{1}{\operatorname{Re}} \Delta u+\nabla p & =f & & \text { in } \Omega \\
\operatorname{div} u & =0 & & \text { in } \Omega
\end{aligned}
$$

together with the slip boundary condition

$$
u \cdot n=0 \quad \text { on } \partial \Omega
$$

To this boundary condition we have to add a condition on the tangential stresses, for instance

$$
n \cdot \sigma(u, p) \tau_{i}=0 \quad \text { on } \partial \Omega, \quad i=2, \ldots, d .
$$

Keywords and phrases. Stokes equations, Navier-Stokes equations, finite elements, slip boundary condition, error estimates.

${ }^{1}$ Universität Bremen, Fachbereich 3, Zentrum für Technomathematik, Postfach 330 440, 28334 Bremen, Germany.

e-mail: baensch@math.uni-bremen.de

${ }^{2}$ Centre for Mathematical Analysis and its Applications, School of Mathematical Sciences, University of Sussex, Falmer, Brighton BN1 9QH, England. e-mail: k.p.deckelnick@sussex.ac.uk 
Here, Re denotes the Reynolds number, $n, \tau_{i}$ the normal and tangential vectors on $\partial \Omega$ and

$$
\sigma_{i, j}=\sigma(u, p)_{i, j}=\frac{1}{\operatorname{Re}} D(u)_{i, j}-p \delta_{i, j}
$$

is the stress tensor with

$$
D(u)_{i, j}=\left(\frac{\partial u_{i}}{\partial x_{j}}+\frac{\partial u_{j}}{\partial x_{i}}\right)
$$

the deformation tensor.

Boundary conditions $(1.3,1.4)$ play an important role in many physical situations, in particular for free boundary problems. We mention:

- coating flows, see for instance $[11,16]$,

- flow in semiconductor melts, see e.g. $[10,17]$.

In contrast to the Stokes and Navier-Stokes equations with Dirichlet boundary condition there seems to be rather few work concerned with numerical analysis of this problem with slip boundary condition. In [20] Verfürth proved error estimates for the discretization of (1.1-1.4) by the popular Taylor-Hood element and a polyhedral approximation $\Omega_{h}$ of the domain $\Omega$. Verfürth gave a non optimal error bound of $\mathcal{O}\left(h^{1 / 2}\right)$ in the $H^{1}$ and $L^{2}$ norm for the velocity and pressure respectively. In $[21,22]$ the same author proposed and analyzed a discretization where the slip boundary condition is enforced in a weak sense by Lagrange multipliers. Numerically, however, in most cases it is more convenient to use a discretization, where the slip boundary condition is incorporated in the ansatz space, see also [2].

The present article therefore analyzes a discretization of this type for the Taylor-Hood element. We improve the result from [20] to the optimal error bound $\mathcal{O}\left(h^{3 / 2}\right)$ for the velocity and the pressure in $H^{1}$ and $L^{2}$ norms respectively for a polyhedral approximation of $\Omega$.

The paper is organized as follows: In Section 2 the finite element formulation and some notations are given. In particular, we introduce a homeomorphism $G_{h}$ which maps the discrete domain $\tilde{\Omega}_{h}$ onto $\Omega$. We prove optimal error estimates for the Stokes problem in Section 3. The key idea is to transform the discrete solution via $G_{h}$ onto the original domain $\Omega$ and to carry out the error analysis on $\Omega$. We thereby avoid error terms which involve integration over the discrete boundary $\partial \tilde{\Omega}_{h}$. In Section 4 optimal error bounds are also obtained for the nonlinear Navier-Stokes equations in case of small Reynolds numbers. Section 5 concludes the article by presenting numerical results.

Remark 1.1. After having finished this paper we got to know about a related recent paper by Knobloch [12]. He considers the Stokes equations allowing both slip and no-slip boundary conditions. For a tetrahedral approximation of $\Omega$ and finite element spaces (including the Taylor-Hood element) satisfying suitable assumptions he obtains optimal orders of convergence. Our technique however is different from his in that we use the transformation $G_{h}$ in order to carry out the analysis on $\Omega$ which simplifies the calculations considerably. A further difference is that we also treat the nonlinear problem and support our analysis by a numerical example.

\section{Finite ElEMENT FORMUlation}

In the following we assume for simplicity that $\Omega$ has no axis of symmetry. This will be needed to insure ellipticity of our problem, see (2.3) below.

In order to derive a variational formulation of $(1.2-1.4)$ the momentum equation in (1.2) is multiplied by a function $v \in H^{1}\left(\Omega ; \mathbb{R}^{3}\right)$ and integrated by parts:

$$
\begin{aligned}
\int_{\Omega}\left(-\frac{1}{\operatorname{Re}} \Delta u+\nabla p\right) \cdot v & =\frac{1}{2 \operatorname{Re}} \int_{\Omega} D(u): D(v)-\int_{\Omega} p \operatorname{div} v-\int_{\partial \Omega} n \cdot \sigma(u, p) v \\
& =\frac{1}{2 \operatorname{Re}} \int_{\Omega} D(u): D(v)-\int_{\Omega} p \operatorname{div} v-\int_{\partial \Omega}(n \cdot \sigma(u, p) n) v \cdot n
\end{aligned}
$$


where the last equality follows from (1.4). Now it is natural to introduce the following bilinear forms:

$$
\begin{aligned}
a(u, v) & :=\frac{1}{2 \operatorname{Re}} \int_{\Omega} D(u): D(v) & & \text { for } u, v \in H^{1}\left(\Omega ; \mathbb{R}^{3}\right) \\
b(v, q) & :=-\int_{\Omega} q \operatorname{div} v & & \text { for } v \in H^{1}\left(\Omega ; \mathbb{R}^{3}\right), \quad q \in L^{2}(\Omega)
\end{aligned}
$$

as well as the function spaces

$$
\begin{aligned}
X & :=\left\{v \in H^{1}\left(\Omega ; \mathbb{R}^{3}\right) \mid v \cdot n=0 \quad \text { on } \partial \Omega\right\} \\
M & :=\left\{q \in L^{2}(\Omega) \mid \int_{\Omega} q=0\right\} .
\end{aligned}
$$

The weak formulation of $(1.2-1.4)$ then reads: find $(u, p) \in X \times M$ such that

$$
\begin{aligned}
a(u, v)+b(v, p) & =(f, v) & & \text { for all } v \in X \\
b(u, q) & =0 & & \text { for all } q \in M
\end{aligned}
$$

where $(f, v)=\int_{\Omega} f v \mathrm{~d} x$ denotes the $L^{2}$ inner product. Note that a weak solution which is smooth is also a solution of (1.2-1.4). Both existence and uniqueness of a weak solution follow from Korn's inequality, see (3.4) below

$$
\|v\|_{H^{1}(\Omega)}^{2} \leq C \int_{\Omega} D(v): D(v) \quad \text { for all } v \in X
$$

the Babuška-Brezzi condition

$$
\inf _{q \in M \backslash\{0\}} \sup _{v \in X \backslash\{0\}} \frac{b(v, q)}{\|v\|_{H^{1}}\|q\|_{L^{2}}} \geq \beta>0
$$

and the general theory of saddle point problems, $c f .[5,9]$.

Regularity properties of $(u, p)$ were studied for instance in [18], in particular we have

$$
\|u\|_{H^{3}(\Omega)}^{2}+\|p\|_{H^{2}(\Omega)}^{2} \leq C\|f\|_{H^{1}(\Omega)}^{2} \quad \text { if } \Omega \text { is of class } C^{4} .
$$

Next, let us denote by $\tilde{\mathcal{T}}_{h}$ a finite set of straight, closed $d$-simplices which triangulates a domain

$$
\bar{\Omega}_{h}=\bigcup_{\tilde{T} \in \tilde{\mathcal{T}}_{h}} \tilde{T}
$$

in such a way that all vertices on $\partial \tilde{\Omega}_{h}$ also lie on $\partial \Omega$. Denote by $h(\tilde{T})=\operatorname{diam}(\tilde{T})$ the diameter of $\tilde{T}$ and by $\rho(\tilde{T})$ the radius of the largest ball inscribed $\tilde{T}$. We make the usual assumption of shape regularity, i.e. for a family of triangulations $\left(\tilde{\mathcal{T}}_{h}\right)_{h}$ we assume that

$$
\sup _{h} \max _{\tilde{T} \in \tilde{\tau_{h}}} \frac{h(\tilde{T})}{\rho(\tilde{T})} \leq \kappa<\infty .
$$


For every $\tilde{T} \in \tilde{\mathcal{T}}_{h}$ there exists an invertible affine mapping

$$
\tilde{F}_{\tilde{T}}: \mathbb{R}^{d} \rightarrow \mathbb{R}^{d}, \quad \tilde{F}_{\tilde{T}}(\hat{x})=A_{\tilde{T}} \hat{x}+b_{\tilde{T}}
$$

which maps the standard $d$-simplex $\hat{T}$ onto $\tilde{T}$. Besides the triangulation $\tilde{\mathcal{T}}_{h}$ which will be used to define the discrete problem and to carry out the practical computations we also introduce an exact triangulation $\mathcal{T}_{h}$ of $\Omega$. The existence of such a triangulation together with the associated interpolation estimates is proved in $[4,13]$. In essence, for every $\tilde{T} \in \tilde{\mathcal{T}}_{h}$ there is a mapping $\Phi_{\tilde{T}} \in C^{3}\left(\hat{T} ; \mathbb{R}^{d}\right)$ such that $F_{\tilde{T}}:=\tilde{F}_{\tilde{T}}+\Phi_{\tilde{T}}$ maps $\hat{T}$ onto a curved $d$-simplex $T \subseteq \bar{\Omega}$ and

$$
\bar{\Omega}=\bigcup_{T \in \mathcal{T}_{h}} T .
$$

Furthermore, the mapping $G_{h}$ which is locally defined by

$$
G_{h \mid \tilde{T}}:=F_{\tilde{T}} \circ \tilde{F}_{\tilde{T}}^{-1}
$$

(see Fig. 1) is a homeomorphism between $\tilde{\Omega}_{h}$ and $\Omega$. The construction in $[4,13]$ also implies that $\Phi_{\tilde{T}}=0$ if $\tilde{T}$ has at most one vertex on $\partial \tilde{\Omega}_{h}$, so that $G_{h} \equiv I$ on all simplices which are disjoint from $\partial \tilde{\Omega}_{h}$. Finally, we have the estimates

$$
\begin{aligned}
& \sup _{x \in \tilde{T}}\left\|\left(D G_{h \mid \tilde{T}}-I\right)(x)\right\| \leq C h(\tilde{T}), \quad\left\|G_{h}\right\|_{H^{3, \infty}(\tilde{T})} \leq C \\
& \sup _{\hat{x} \in \hat{T}}\left\|D F_{\tilde{T}}(\hat{x})\right\| \leq C\left\|A_{\tilde{T}}\right\|, \quad \sup _{x \in T}\left\|D F_{\tilde{T}}^{-1}(x)\right\| \leq C\left\|A_{\tilde{T}}^{-1}\right\| \\
& c_{1}\left|\operatorname{det} A_{\tilde{T}}\right| \leq\left|\operatorname{det} D F_{\tilde{T}}(\hat{x})\right| \leq c_{2}\left|\operatorname{det} A_{\tilde{T}}\right|, \quad \hat{x} \in \hat{T}
\end{aligned}
$$

for all $\tilde{T} \in \tilde{\mathcal{T}}_{h}$. In particular, $v \in H^{1}(\Omega)$ if and only if $v \circ G_{h} \in H^{1}\left(\tilde{\Omega}_{h}\right)$ and

$$
c_{3}\|v\|_{H^{1}(\Omega)} \leq\left\|v \circ G_{h}\right\|_{H^{1}\left(\tilde{\Omega}_{h}\right)} \leq c_{4}\|v\|_{H^{1}(\Omega)} .
$$

Let us turn to the definition of the finite element spaces which we shall use. We denote by $\mathcal{N}_{h}$ the union of the set of all vertices of $\tilde{\mathcal{T}}_{h}$ with the set of all midpoints of edges of $d$-simplices in $\tilde{\mathcal{T}}_{h}$. Then we define

$$
X_{h}:=\left\{v_{h} \in C^{0}\left(\bar{\Omega}_{h} ; \mathbb{R}^{d}\right) \mid v_{h \mid \tilde{T}} \in \mathcal{P}_{2}(\tilde{T})^{d}, v_{h}(p) \cdot n\left(G_{h}(p)\right)=0 \forall p \in \mathcal{N}_{h} \cap \partial \tilde{\Omega}_{h}\right\}
$$

and

$$
M_{h}:=\left\{q_{h} \in C^{0}\left(\bar{\Omega}_{h}\right) \mid q_{h \mid \tilde{T}} \in \mathcal{P}_{1}(\tilde{T}), \int_{\tilde{\Omega}_{h}} q_{h}=0\right\}
$$

that is we use the so called Taylor-Hood element and enforce the slip boundary condition pointwise in all boundary vertices and midpoints. Note that the normal $n$ appearing in the definition of $X_{h}$ is the normal to the domain $\Omega$. Defining $a_{h}: X_{h} \times X_{h} \rightarrow \mathbb{R}, b_{h}: X_{h} \times M_{h} \rightarrow \mathbb{R}$ by

$$
\begin{aligned}
a_{h}\left(u_{h}, v_{h}\right) & :=\frac{1}{2 \operatorname{Re}} \int_{\tilde{\Omega}_{h}} D\left(u_{h}\right): D\left(v_{h}\right) \\
b_{h}\left(v_{h}, q_{h}\right) & :=-\int_{\tilde{\Omega}_{h}} q_{h} \operatorname{div} v_{h}
\end{aligned}
$$




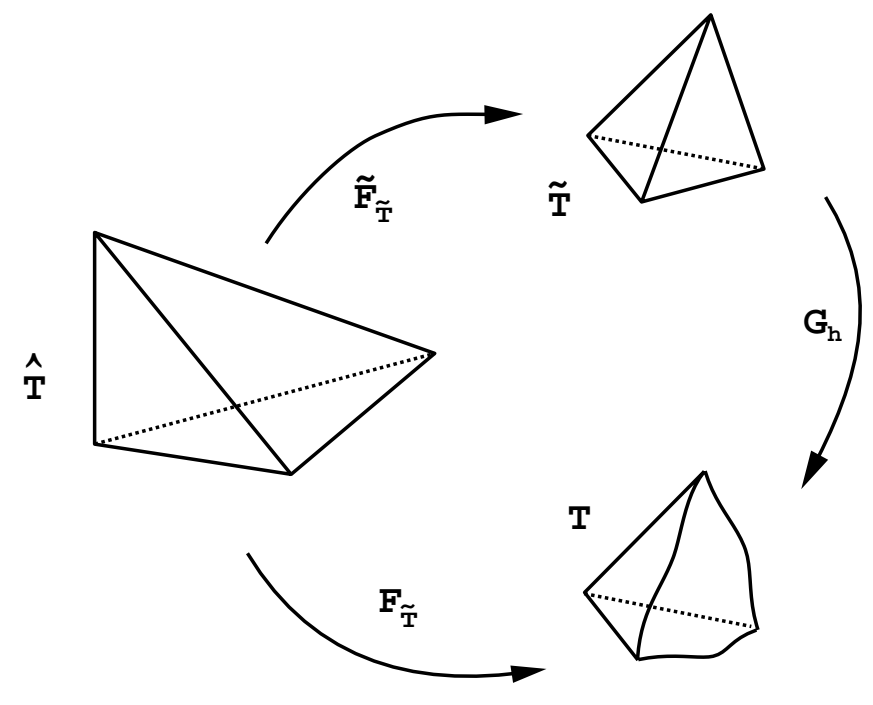

FiguRE 1. $\hat{T}, \tilde{T}$ and $T$ at the boundary.

the discrete analogy of $(2.2)$ reads: find $\left(u_{h}, p_{h}\right) \in X_{h} \times M_{h}$ such that

$$
\begin{aligned}
a_{h}\left(u_{h}, v_{h}\right)+b_{h}\left(v_{h}, p_{h}\right) & =\int_{\tilde{\Omega}_{h}} \tilde{f} \cdot v_{h} & & \text { for all } v_{h} \in X_{h} \\
b_{h}\left(u_{h}, q_{h}\right) & =0 & & \text { for all } q_{h} \in M_{h}
\end{aligned}
$$

where $\tilde{f}:=f \circ G_{h}$. It follows from [20] that (2.7) has a unique solution for all $0<h \leq h_{0}$ with $h_{0}$ small enough.

Remark 2.1. Since the Babuška-Brezzi condition can be proved for the Taylor-Hood element without using an inverse estimate, see [15], we do not assume quasi uniformity for the triangulation $\tilde{\mathcal{T}}_{h}$. Thus our analysis is valid also in the case of adaptively refined meshes.

Remark 2.2. We have introduced $\tilde{f}$ above because we do not want to overburden the error analysis with the approximation of $\int_{\Omega} f \cdot v_{h}$. This can be done with the help of a suitable quadrature rule under appropriate regularity assumptions on $f$ (cf. [7]: Chap. 4, Sect. 4.1).

\section{Proof of the ERror estimate}

One problem of the error analysis lies in the fact that $(u, p)$ and $\left(u_{h}, p_{h}\right)$ are defined on different spaces. To overcome this difficulty we assign to each $\left(v_{h}, q_{h}\right) \in X_{h} \times M_{h}$ the pair

$$
\left(\bar{v}_{h}, \bar{q}_{h}\right):=\left(v_{h} \circ G_{h}^{-1}, q_{h} \circ G_{h}^{-1}-\frac{1}{|\Omega|} \int_{\Omega} q_{h} \circ G_{h}^{-1}\right) \in H^{1}\left(\Omega ; \mathbb{R}^{d}\right) \times M .
$$

Note that in general $\bar{v}_{h} \notin X$ since $v_{h} \cdot\left(n \circ G_{h}\right)$ only vanishes in the points of $\mathcal{N}_{h}$. Inserting $\bar{v}_{h}$ into (2.1) we get

$$
a\left(u, \bar{v}_{h}\right)+b\left(\bar{v}_{h}, p\right)=\int_{\Omega} f \cdot \bar{v}_{h}+\int_{\partial \Omega}(n \sigma(u, p) n) \bar{v}_{h} \cdot n, \quad v_{h} \in X_{h} .
$$


Combining this identity with (2.7) we obtain the following error relation for $\left(u-\bar{u}_{h}, p-\bar{p}_{h}\right)$ :

$$
\begin{aligned}
a\left(u-\bar{u}_{h}, \bar{v}_{h}\right)+b\left(\bar{v}_{h}, p-\bar{p}_{h}\right)= & a_{h}\left(u_{h}, v_{h}\right)-a\left(\bar{u}_{h}, \bar{v}_{h}\right)+b_{h}\left(v_{h}, p_{h}\right)-b\left(\bar{v}_{h}, \bar{p}_{h}\right) \\
& +\int_{\Omega} f \cdot \bar{v}_{h}-\int_{\tilde{\Omega}_{h}} \tilde{f} \cdot v_{h}+\int_{\partial \Omega}(n \sigma(u, p) n) \bar{v}_{h} \cdot n \\
b\left(u-\bar{u}_{h}, \bar{q}_{h}\right)= & b_{h}\left(u_{h}, q_{h}\right)-b\left(\bar{u}_{h}, \bar{q}_{h}\right)
\end{aligned}
$$

for all $v_{h} \in X_{h}, q_{h} \in M_{h}$.

Before we can apply well-known results on the approximation of saddle point problems we need suitable discrete analogies of the ellipticity condition and the Babuška-Brezzi condition.

The following lemma will be very helpful in the subsequent analysis.

Lemma 3.1. There exists $h_{1}>0$ such that for all $0<h \leq h_{1}$ and all $v_{h} \in X_{h}$

$$
\left\|\bar{v}_{h} \cdot n\right\|_{L^{2}(\partial \Omega)} \leq \operatorname{ch}^{\frac{3}{2}}\left\|\bar{v}_{h}\right\|_{H^{1}(\Omega)} .
$$

Proof. Let us denote by $\mathcal{T}_{h}^{\partial}$ the set of all simplices in $\mathcal{T}_{h}$ which have a $(d-1)$-face on $\partial \Omega$. The transformation rule and (2.6) imply

$$
\int_{\partial \Omega}\left|\bar{v}_{h} \cdot n\right|^{2}=\sum_{T \in \mathcal{T}_{h}^{\partial}} \int_{\partial \Omega \cap T}\left|\left(v_{h} \circ G_{h}^{-1}\right) \cdot n\right|^{2} \leq c \sum_{T \in \mathcal{T}_{h}^{\partial}} \int_{\partial \tilde{\Omega}_{h} \cap \tilde{T}}\left|v_{h} \cdot\left(n \circ G_{h}\right)\right|^{2} .
$$

Let us fix $T \in \mathcal{T}_{h}^{\partial}$ and put $\tilde{\Gamma}:=\partial \tilde{\Omega}_{h} \cap \tilde{T}$. In view of the definition of $X_{h}$ we have $I_{h}^{2}\left(v_{h \mid \tilde{\Gamma}} \cdot\left(n \circ G_{h}\right)_{\mid \tilde{\Gamma}}\right) \equiv 0$ where $I_{h}^{2}$ denotes the Lagrange interpolation operator for polynomials of degree two. If we apply a well-known interpolation result ( $c f$. [7]) we get

$$
\begin{aligned}
\int_{\tilde{\Gamma}}\left|v_{h} \cdot\left(n \circ G_{h}\right)\right|^{2} & =\int_{\tilde{\Gamma}}\left|v_{h} \cdot\left(n \circ G_{h}\right)-I_{h}^{2}\left(v_{h} \cdot\left(n \circ G_{h}\right)\right)\right|^{2} \\
& \leq \operatorname{ch}(\tilde{T})^{6} \int_{\tilde{\Gamma}}\left|D^{3}\left(\left(v_{h} \cdot\left(n \circ G_{h}\right)\right)_{\mid \tilde{\Gamma}}\right)\right|^{2} .
\end{aligned}
$$

Observing that $v_{h \mid \tilde{\Gamma}} \in P_{2}(\tilde{\Gamma})$ as well as $\partial \Omega \in C^{4}$ and using (2.6) we may estimate

$$
\begin{aligned}
\int_{\tilde{\Gamma}}\left|D^{3}\left(v_{h} \cdot\left(n \circ G_{h}\right)\right)\right|^{2} & \leq c\left\|v_{h}\right\|_{H^{2}(\tilde{\Gamma})}^{2}=c\left(\left\|v_{h}\right\|_{L^{2}(\tilde{\Gamma})}^{2}+\left\|D v_{h}\right\|_{H^{1}(\tilde{\Gamma})}^{2}\right) \\
& \leq \operatorname{ch}(\tilde{T})^{-1}\left\|v_{h}\right\|_{L^{2}(\tilde{T})}^{2}+\operatorname{ch}(\tilde{T})^{-2}\left\|v_{h}\right\|_{H^{1}(\tilde{\Gamma})}^{2} \\
& \leq \operatorname{ch}(\tilde{T})^{-1}\left\|v_{h}\right\|_{L^{2}(\tilde{T})}^{2}+\operatorname{ch}(\tilde{T})^{-3}\left\|v_{h}\right\|_{H^{1}(\tilde{T})}^{2} \\
& \leq \operatorname{ch}(\tilde{T})^{-3}\left\|v_{h}\right\|_{H^{1}(\tilde{T})}^{2}
\end{aligned}
$$

in view of the inverse estimates

$$
\left\|v_{h}\right\|_{L^{2}(\tilde{\Gamma})} \leq \operatorname{ch}(\tilde{T})^{-\frac{1}{2}}\left\|v_{h}\right\|_{L^{2}(\tilde{T})}, \quad\left\|D v_{h}\right\|_{L^{2}(\tilde{\Gamma})} \leq \operatorname{ch}(\tilde{T})^{-1}\left\|v_{h}\right\|_{L^{2}(\tilde{\Gamma})}
$$


In conclusion

$$
\int_{\partial \Omega}\left|\bar{v}_{h} \cdot n\right|^{2} \leq c \sum_{T \in \mathcal{T}_{h}^{\partial}} h(\tilde{T})^{3}\left\|v_{h}\right\|_{H^{1}(\tilde{T})}^{2} \leq c h^{3} \sum_{T \in \mathcal{T}_{h}^{\partial}}\left\|\bar{v}_{h}\right\|_{H^{1}(T)}^{2} \leq c h^{3}\left\|\bar{v}_{h}\right\|_{H^{1}(\Omega)}^{2}
$$

and the lemma is proved.

Note that we made use of the inverse estimate only locally, hence we do not need a quasiuniformity assumption on the triangulation.

The above result should be compared to Lemma 3.1 in [20] where the author obtained the boundary estimate

$$
\left\|v_{h} \cdot n_{h}\right\|_{L^{2}\left(\partial \tilde{\Omega}_{h}\right)} \leq c h^{\frac{1}{2}}\left\|v_{h}\right\|_{H^{1}\left(\tilde{\Omega}_{h}\right)}, \quad v_{h} \in X_{h}
$$

which then led to the suboptimal error bound $\mathcal{O}\left(h^{\frac{1}{2}}\right)$. By employing the transformation $G_{h}$ we are able to improve this estimate and this will turn out to be crucial in the proof of the optimal convergence rate.

The next lemma estimates the effect of the transformation on the bilinear forms.

Lemma 3.2. There exists $h_{2}>0$ such that for all $0<h \leq h_{2}$ and for all $v_{h}, w_{h} \in X_{h}, q_{h} \in M_{h}$

$$
\begin{aligned}
\left|a\left(\bar{v}_{h}, \bar{w}_{h}\right)-a_{h}\left(v_{h}, w_{h}\right)\right| & \leq c h\left(\sum_{T \cap \partial \Omega \neq \emptyset}\left\|\bar{v}_{h}\right\|_{H^{1}(T)}^{2}\right)^{\frac{1}{2}}\left(\sum_{T \cap \partial \Omega \neq \emptyset}\left\|\bar{w}_{h}\right\|_{H^{1}(T)}^{2}\right)^{\frac{1}{2}} \\
\left|b\left(\bar{v}_{h}, \bar{q}_{h}\right)-b_{h}\left(v_{h}, q_{h}\right)\right| & \leq c^{\frac{3}{2}}\left\|\bar{v}_{h}\right\|_{H^{1}(\Omega)}\left\|\bar{q}_{h}\right\|_{L^{2}(\Omega)}+c h\left(\sum_{T \cap \partial \Omega \neq \emptyset}\left\|\bar{v}_{h}\right\|_{H^{1}(T)}^{2}\right)^{\frac{1}{2}}\left(\sum_{T \cap \partial \Omega \neq \emptyset}\left\|\bar{q}_{h}\right\|_{L^{2}(T)}^{2}\right)^{\frac{1}{2}}
\end{aligned}
$$

Proof. Note first that $G_{h} \equiv I$ on all simplices which are disjoint from $\partial \tilde{\Omega}_{h}$. Then the first assertion can be proved in the same way as Lemma 8(ii) in [13].

In order to show (b) we first estimate $\int_{\Omega} q_{h} \circ G_{h}^{-1}$. Observing that $\int_{\tilde{\Omega}_{h}} q_{h}=0$ we get

$$
\begin{aligned}
\int_{\Omega} q_{h} \circ G_{h}^{-1} & =\sum_{T \cap \partial \Omega \neq \emptyset} \int_{T} q_{h} \circ G_{h}^{-1}+\sum_{T \cap \partial \Omega=\emptyset} \int_{T} q_{h} \circ G_{h}^{-1} \\
& =\sum_{T \cap \partial \Omega \neq \emptyset} \int_{T} q_{h} \circ G_{h}^{-1}+\sum_{T \cap \partial \Omega=\emptyset} \int_{\tilde{T}} q_{h} \\
& =\sum_{T \cap \partial \Omega \neq \emptyset}\left(\int_{\tilde{T}} q_{h}\left|\operatorname{det} G_{h}\right|-\int_{\tilde{T}} q_{h}\right)
\end{aligned}
$$

in view of the transformation rule. From (2.6) and Hölder's inequality we obtain

$$
\begin{aligned}
\left|\int_{\Omega} q_{h} \circ G_{h}^{-1}\right| & \leq c \sum_{T \cap \partial \Omega \neq \emptyset} h(\tilde{T}) \int_{\tilde{T}}\left|q_{h}\right| \leq c \sum_{T \cap \partial \Omega \neq \emptyset} h(\tilde{T})^{\frac{d}{2}+1}\left\|q_{h}\right\|_{L^{2}(\tilde{T})} \\
& \leq c\left(\sum_{T \cap \partial \Omega \neq \emptyset} h(\tilde{T})^{d+2}\right)^{\frac{1}{2}}\left\|q_{h}\right\|_{L^{2}\left(\tilde{\Omega}_{h}\right)} \leq c h^{\frac{3}{2}}\left\|q_{h} \circ G_{h}^{-1}\right\|_{L^{2}(\Omega)} \\
& \leq c h^{\frac{3}{2}}\left\|\bar{q}_{h}\right\|_{L^{2}(\Omega)}+c h^{\frac{3}{2}}\left|\int_{\Omega} q_{h} \circ G_{h}^{-1}\right|
\end{aligned}
$$


since $q_{h} \circ G_{h}^{-1}=\bar{q}_{h}+\frac{1}{|\Omega|} \int_{\Omega} q_{h} \circ G_{h}^{-1}$ and

$$
\sum_{T \cap \partial \Omega \neq \emptyset} h(\tilde{T})^{d-1} \leq c \mathcal{H}^{d-1}\left(\partial \tilde{\Omega}_{h}\right) \leq c \mathcal{H}^{d-1}(\partial \Omega)
$$

with $\mathcal{H}^{d-1}$ the $(d-1)$-dimensional Hausdorff measure. Summarizing the above considerations we get for sufficiently small $h_{2}$

$$
\left|\int_{\Omega} q_{h} \circ G_{h}^{-1}\right| \leq \operatorname{ch}^{\frac{3}{2}}\left\|\bar{q}_{h}\right\|_{L^{2}(\Omega)}
$$

and therefore

$$
\left|b\left(\bar{v}_{h}, \bar{q}_{h}\right)-b_{h}\left(v_{h}, q_{h}\right)\right| \leq\left|b\left(\bar{v}_{h}, q_{h} \circ G_{h}^{-1}\right)-b_{h}\left(v_{h}, q_{h}\right)\right|+c\left\|\bar{v}_{h}\right\|_{H^{1}(\Omega)}\left|\int_{\Omega} q_{h} \circ G_{h}^{-1}\right| .
$$

Estimating the first term again similarly as in Lemma 8(i) in [13] the result follows from (3.3).

Next we obtain discrete versions of the conditions $(2.3,2.4)$.

Lemma 3.3. There exists $h_{3}>0$ such that for all $0<h \leq h_{3}$

$$
\begin{aligned}
& a\left(\bar{v}_{h}, \bar{v}_{h}\right) \geq c_{0}\left\|\bar{v}_{h}\right\|_{H^{1}(\Omega)}^{2}, \quad v_{h} \in X_{h} \\
& \inf _{q_{h} \in M_{h} \backslash\{0\}} \sup _{v_{h} \in X_{h} \backslash\{0\}} \frac{b\left(\bar{v}_{h}, \bar{q}_{h}\right)}{\left\|\bar{v}_{h}\right\|_{H^{1}(\Omega)}\left\|\bar{q}_{h}\right\|_{L^{2}(\Omega)}} \geq \beta^{\prime}>0 .
\end{aligned}
$$

Proof. (a) Korn's second inequality (cf. [14]) together with the Poincaré-Morrey inequality (cf. [21]) implies

$$
\|v\|_{H^{1}(\Omega)}^{2} \leq c\left(\int_{\Omega} D(v): D(v)+\|v\|_{L^{2}(\Omega)}^{2}\right) \leq c\left(\int_{\Omega} D(v): D(v)+\int_{\partial \Omega}|v \cdot n|^{2}\right)
$$

for all $v \in H^{1}\left(\Omega ; \mathbb{R}^{d}\right)$. Applying this estimate to $\bar{v}_{h}$ and using Lemma 3.1 we arrive at

$$
\left\|\bar{v}_{h}\right\|_{H^{1}(\Omega)}^{2} \leq c a\left(\bar{v}_{h}, \bar{v}_{h}\right)+c h^{3}\left\|\bar{v}_{h}\right\|_{H^{1}(\Omega)}^{2}
$$

which gives (a).

(b) According to [20] the following condition is valid:

$$
\inf _{q_{h} \in M_{h} \backslash\{0\}} \sup _{v_{h} \in X_{h} \backslash\{0\}} \frac{b_{h}\left(v_{h}, q_{h}\right)}{\left\|v_{h}\right\|_{H^{1}\left(\tilde{\Omega}_{h}\right)}\left\|q_{h}\right\|_{L^{2}\left(\tilde{\Omega}_{h}\right)}} \geq \beta>0 .
$$

The assertion now follows from Lemma $3.2 \mathrm{~b}$ if we observe that $\left\|\bar{v}_{h}\right\|_{H^{1}(\Omega)},\left\|v_{h}\right\|_{H^{1}\left(\tilde{\Omega}_{h}\right)}$ and $\left\|\bar{q}_{h}\right\|_{L^{2}(\Omega)},\left\|q_{h}\right\|_{L^{2}\left(\tilde{\Omega}_{h}\right)}$ are equivalent norms for $v_{h}, q_{h}$ respectively.

Now we are in position to prove the main result of this paper.

Theorem 3.4. Let $(u, p)$ be the solution of (1.2-1.4) and $\left(u_{h}, p_{h}\right)$ the solution of (2.2). Then there exists $h_{4}>0$ such that for all $0<h \leq h_{4}$

$$
\left\|u-\bar{u}_{h}\right\|_{H^{1}(\Omega)}+\left\|p-\bar{p}_{h}\right\|_{L^{2}(\Omega)} \leq \operatorname{ch}^{\frac{3}{2}}\|f\|_{H^{1}(\Omega)} .
$$


Proof. As we do not have $\left\{\bar{v}_{h} \mid v_{h} \in X_{h}\right\} \subset X$ we consider both spaces as subspaces of $H^{1}\left(\Omega, \mathbb{R}^{d}\right)$. From Lemma 3.3 we infer

$$
\begin{aligned}
a\left(\bar{v}_{h}, \bar{v}_{h}\right) & \geq c_{0}\left\|\bar{v}_{h}\right\|_{H^{1}(\Omega)}^{2}, \quad \text { for all } v_{h} \in X_{h} \\
\sup _{v_{h} \in X_{h} \backslash\{0\}} \frac{b\left(\bar{v}_{h}, \bar{q}_{h}\right)}{\left\|\bar{v}_{h}\right\|_{H^{1}(\Omega)}} & \geq \beta^{\prime}\left\|\bar{q}_{h}\right\|_{L^{2}(\Omega)}, \quad \text { for all } q_{h} \in M_{h} .
\end{aligned}
$$

Using the techniques in Section II.2 in [5] we conclude from the error relation $(3.1,3.5)$

$$
\left\|u-\bar{u}_{h}\right\|_{H^{1}(\Omega)}+\left\|p-\bar{p}_{h}\right\|_{L^{2}(\Omega)} \leq c\left(\inf _{v_{h} \in X_{h}}\left\|u-\bar{v}_{h}\right\|_{H^{1}(\Omega)}+\inf _{q_{h} \in M_{h}}\left\|p-\bar{q}_{h}\right\|_{L^{2}(\Omega)}+\sum_{i=1}^{5} M_{i, h}\right)
$$

where

$$
\begin{aligned}
M_{1, h} & =\sup _{v_{h} \in X_{h} \backslash\{0\}} \frac{\left|a_{h}\left(u_{h}, v_{h}\right)-a\left(\bar{u}_{h}, \bar{v}_{h}\right)\right|}{\left\|\bar{v}_{h}\right\|_{H^{1}(\Omega)}} \\
M_{2, h} & =\sup _{v_{h} \in X_{h} \backslash\{0\}} \frac{\left|b_{h}\left(v_{h}, p_{h}\right)-b\left(\bar{v}_{h}, \bar{p}_{h}\right)\right|}{\left\|\bar{v}_{h}\right\|_{H^{1}(\Omega)}} \\
M_{3, h} & =\sup _{v_{h} \in X_{h} \backslash\{0\}} \frac{\left|\int_{\Omega} f \cdot \bar{v}_{h}-\int_{\tilde{\Omega}_{h}} \tilde{f} \cdot v_{h}\right|}{\left\|\bar{v}_{h}\right\|_{H^{1}(\Omega)}} \\
M_{4, h} & =\sup _{v_{h} \in X_{h} \backslash\{0\}} \frac{\left|\int_{\partial \Omega}(n \sigma(u, p) n) \bar{v}_{h} \cdot n\right|}{\left\|\bar{v}_{h}\right\|_{H^{1}(\Omega)}} \\
M_{5, h} & =\sup _{q_{h} \in M_{h} \backslash\{0\}} \frac{\left|b_{h}\left(u_{h}, q_{h}\right)-b\left(\bar{u}_{h}, \bar{q}_{h}\right)\right|}{\left\|\bar{q}_{h}\right\|_{L^{2}(\Omega)}} .
\end{aligned}
$$

In the following we estimate the various terms occurring on the right hand side of (3.6).

To begin, let us denote by $I_{h}^{2}$ the usual Lagrange interpolation operator on $\tilde{\Omega}_{h}$ for polynomials of degree two and define $w_{h}:=I_{h}^{2}\left(u \circ G_{h}\right)$. Clearly, $w_{h} \in X_{h}$ and

$$
\left\|u \circ G_{h}-w_{h}\right\|_{H^{1}(\tilde{T})} \leq \operatorname{ch}(\tilde{T})^{2}\left\|D^{3}\left(u \circ G_{h}\right)\right\|_{L^{2}(\tilde{T})}, \quad \tilde{T} \in \tilde{\mathcal{T}}_{h} .
$$

Transforming to $\Omega$ and using $(2.5,2.6)$ we get

$$
\begin{aligned}
\left\|u-\bar{w}_{h}\right\|_{H^{1}(\Omega)} & =\left(\sum_{T \in \mathcal{T}_{h}}\left\|u-\bar{w}_{h}\right\|_{H^{1}(T)}^{2}\right)^{\frac{1}{2}} \leq c\left(\sum_{\tilde{T} \in \tilde{\mathcal{T}}_{h}}\left\|u \circ G_{h}-w_{h}\right\|_{H^{1}(\tilde{T})}^{2}\right)^{\frac{1}{2}} \\
& \leq \operatorname{ch}^{2}\left(\sum_{\tilde{T} \in \tilde{\mathcal{T}}_{h}}\left\|D^{3}\left(u \circ G_{h}\right)\right\|_{L^{2}(\tilde{T})}^{2}\right)^{\frac{1}{2}} \leq c h^{2}\|u\|_{H^{3}(\Omega)} \\
& \leq \operatorname{ch}^{2}\|f\|_{H^{1}(\Omega)} .
\end{aligned}
$$

Arguing similarly for the pressure we arrive at

$$
\inf _{v_{h} \in X_{h}}\left\|u-\bar{v}_{h}\right\|_{H^{1}(\Omega)}+\inf _{q_{h} \in M_{h}}\left\|p-\bar{q}_{h}\right\|_{L^{2}(\Omega)} \leq c h^{2}\|f\|_{H^{1}(\Omega)} .
$$


Next, Lemma 3.2 gives

$$
\begin{aligned}
M_{1, h} & \leq c h\left(\sum_{T \cap \partial \Omega \neq \emptyset}\left\|\bar{u}_{h}\right\|_{H^{1}(T)}^{2}\right)^{\frac{1}{2}} \\
& \leq c h\left\|u-\bar{u}_{h}\right\|_{H^{1}(\Omega)}+c h\left(\sum_{T \cap \partial \Omega \neq \emptyset}\|u\|_{H^{1}(T)}^{2}\right)^{\frac{1}{2}} \\
& \leq c h\left\|u-\bar{u}_{h}\right\|_{H^{1}(\Omega)}+c\|u\|_{C^{1}(\bar{\Omega})} h\left(\sum_{T \cap \partial \Omega \neq \emptyset} h(T)^{3}\right)^{\frac{1}{2}} \\
& \leq c h\left\|u-\bar{u}_{h}\right\|_{H^{1}(\Omega)}+\operatorname{ch}^{\frac{3}{2}}\|f\|_{H^{1}(\Omega)}
\end{aligned}
$$

where we used the continuous embedding $H^{3}(\Omega) \hookrightarrow C^{1}(\bar{\Omega}),(3.2)$ and (2.5). Similarly

$$
M_{2, h} \leq \operatorname{ch}\left\|p-\bar{p}_{h}\right\|_{L^{2}(\Omega)}+\operatorname{ch}^{\frac{3}{2}}\|f\|_{H^{1}(\Omega)} .
$$

From the definition of $\tilde{f}$ and the fact that $G_{h} \equiv I$ on all simplices which are disjoint from $\partial \tilde{\Omega}_{h}$ we infer

$$
\begin{aligned}
\left|\int_{\Omega} f \cdot \bar{v}_{h}-\int_{\tilde{\Omega}_{h}} \tilde{f} \cdot v_{h}\right| & =\left|\sum_{\tilde{T} \cap \partial \tilde{\Omega}_{h} \neq \emptyset} \int_{\tilde{T}}\left(f \circ G_{h}\right) \cdot v_{h}\left(\left|\operatorname{det} D G_{h}\right|-1\right)\right| \\
& \leq \operatorname{ch} \sum_{\tilde{T} \cap \partial \tilde{\Omega}_{h} \neq \emptyset}\left\|f \circ G_{h}\right\|_{L^{2}(\tilde{T})}\left\|v_{h}\right\|_{L^{2}(\tilde{T})} \\
& \leq \operatorname{ch}\|f\|_{L^{2}\left(B_{\gamma h}(\partial \Omega) \cap \Omega\right)}\left\|\bar{v}_{h}\right\|_{L^{2}\left(B_{\gamma h}(\partial \Omega) \cap \Omega\right) .}
\end{aligned}
$$

In view of the embedding $H^{1}(\Omega) \hookrightarrow L^{4}(\Omega)$ we obtain

$$
\|f\|_{L^{2}\left(B_{\gamma h}(\partial \Omega) \cap \Omega\right)} \leq\left(\mathcal{L}^{d}\left(B_{\gamma h}(\partial \Omega) \cap \Omega\right)\right)^{\frac{1}{4}}\|f\|_{L^{4}(\Omega)} \leq c h^{\frac{1}{4}}\|f\|_{H^{1}(\Omega)}
$$

where $\mathcal{L}^{d}$ denotes the $d$-dimensional Lebesgue measure.

Using the same argument for $\bar{v}_{h}$ we arrive at

$$
\left|\int_{\Omega} f \cdot \bar{v}_{h}-\int_{\tilde{\Omega}_{h}} \tilde{f} \cdot v_{h}\right| \leq c h^{\frac{3}{2}}\|f\|_{H^{1}(\Omega)}\left\|\bar{v}_{h}\right\|_{H^{1}(\Omega)}
$$

for all $v_{h} \in X_{h}$ which implies

$$
M_{3, h} \leq \operatorname{ch}^{\frac{3}{2}}\|f\|_{H^{1}(\Omega)} .
$$

Employing Lemma 3.1 we get

$$
\begin{aligned}
\left|\int_{\partial \Omega}(n \sigma(u, p) n) \bar{v}_{h} \cdot n\right| & \leq c\left(\|u\|_{C^{1}(\bar{\Omega})}+\|p\|_{C^{0}(\bar{\Omega})}\right)\left\|\bar{v}_{h} \cdot n\right\|_{L^{2}(\partial \Omega)} \\
& \leq c h^{\frac{3}{2}}\|f\|_{H^{1}(\Omega)}\left\|\bar{v}_{h}\right\|_{H^{1}(\Omega)}
\end{aligned}
$$

and therefore

$$
M_{4, h} \leq \operatorname{ch}^{\frac{3}{2}}\|f\|_{H^{1}(\Omega)} .
$$


Finally, it follows in the same way as above

$$
M_{5, h} \leq c h\left\|u-\bar{u}_{h}\right\|_{H^{1}(\Omega)}+\operatorname{ch}^{\frac{3}{2}}\|f\|_{H^{1}(\Omega)} .
$$

Combining the above estimates and choosing $h_{4}$ small enough the result follows.

\section{ERror estimates For the NONLINEAR PROBlem}

In this section we extend our error analysis to the Navier-Stokes problem $(1.1,1.3,1.4)$. The discrete problem now reads: find $\left(u_{h}, p_{h}\right) \in X_{h} \times M_{h}$ such that

$$
\begin{aligned}
a_{h}\left(u_{h}, v_{h}\right)+b_{h}\left(v_{h}, p_{h}\right)+N_{h}\left(u_{h}, u_{h}, v_{h}\right) & =\int_{\tilde{\Omega}_{h}} \tilde{f} \cdot v_{h} & & \text { for all } v_{h} \in X_{h} \\
b_{h}\left(u_{h}, q_{h}\right) & =0 & & \text { for all } q_{h} \in M_{h} .
\end{aligned}
$$

Here, $X_{h}, M_{h}, a_{h}$ and $b_{h}$ are the same as in Section 2 while $N_{h}$ is defined by

$$
N_{h}(u, v, w):=\frac{1}{2} \int_{\tilde{\Omega}_{h}}(((u \cdot \nabla) v) w-((u \cdot \nabla) w) v), \quad u, v, w \in H^{1}\left(\tilde{\Omega}_{h} ; \mathbb{R}^{d}\right)
$$

It is well-known that $(1.1,1.3,1.4)$ has a unique solution $(u, p) \in H^{3}\left(\Omega ; \mathbb{R}^{d}\right) \times H^{2}(\Omega)$ provided

$$
(\operatorname{Re})^{2}\|f\|_{L^{2}(\Omega)}<\bar{c}(\Omega)
$$

with some constant $\bar{c}(\Omega)>0$ depending on the domain $\Omega$.

Furthermore,

$$
\|u\|_{H^{1}(\Omega)} \leq c \operatorname{Re}\|f\|_{L^{2}(\Omega)} .
$$

In what follows we assume that (4.2) holds. We shall use a quantified version of Newton's method in order to derive an error bound for the velocity.

Theorem 4.1. Let $X, Y$ be Banach spaces, $u_{0} \in X, R>0$ and $F \in C^{1}\left(B_{R}\left(u_{0}\right), Y\right)$. Assume that DF( $\left.u_{0}\right)$ is an isomorphism of $X$ onto $Y$ with $\left\|D F\left(u_{0}\right)^{-1}\right\|_{L(Y, X)} \leq \gamma$, that $\left\|D F\left(u_{0}\right)^{-1} F\left(u_{0}\right)\right\|_{X} \leq \epsilon$ and that

$$
\|D F(u)-D F(v)\|_{L(X, Y)} \leq K\|u-v\|_{X} \quad \text { for all } u, v \in B_{R}\left(u_{0}\right)
$$

If

$$
2 \epsilon<R, \quad 2 K \epsilon \gamma<1
$$

then the problem $F(u)=0$ has a unique solution $u^{*} \in \overline{B_{2 \epsilon}\left(u_{0}\right)}$.

Proof. See Theorem 15.6 of [8].

Let us now apply the above result to our situation. We set $X=Y=H^{1}\left(\Omega ; \mathbb{R}^{d}\right)$. Let $T_{h} \in L\left(X_{h}^{\prime}, X_{h}\right)$ be the discrete Stokes operator which assigns to every $g_{h} \in X_{h}^{\prime}$ the velocity $u_{h} \in H^{1}\left(\tilde{\Omega}_{h}, \mathbb{R}^{d}\right)$ of the unique solution $\left(u_{h}, p_{h}\right) \in X_{h} \times M_{h}$ of

$$
\begin{aligned}
a_{h}\left(u_{h}, v_{h}\right)+b_{h}\left(v_{h}, p_{h}\right) & =\left\langle g_{h}, v_{h}\right\rangle & & \text { for all } v_{h} \in X_{h} \\
b_{h}\left(u_{h}, q_{h}\right) & =0 & & \text { for all } q_{h} \in M_{h} .
\end{aligned}
$$


Here, $\langle.,$.$\rangle denotes the duality between X_{h}^{\prime}$ and $X_{h}$. We define the mapping $F_{h}: H^{1}\left(\Omega, \mathbb{R}^{d}\right) \rightarrow H^{1}\left(\Omega, \mathbb{R}^{d}\right)$ as follows: for a given $v \in H^{1}\left(\Omega, \mathbb{R}^{d}\right)$ let

$$
w_{h}=T_{h}\left(f \circ G_{h}-N_{h}\left(v \circ G_{h}, v \circ G_{h}, \cdot\right)\right)
$$

and set

$$
F_{h}(v):=v-w_{h} \circ G_{h}^{-1}=v-\bar{w}_{h} .
$$

Clearly, $u_{h}$ is a solution of (4.1) if and only if $F_{h}\left(\bar{u}_{h}\right)=0$.

Now we are in position to formulate the main result of this section.

Theorem 4.2. Let $(u, p)$ be the solution of $(1.1,1.3,1.4)$. If Re $\|f\|_{L^{2}(\Omega)}$ is sufficiently small, there exists $h_{5}>0$ such that for all $0<h \leq h_{5}$ (4.1) has a unique solution $\left(u_{h}, p_{h}\right)$ which satisfies

$$
\left\|u-\bar{u}_{h}\right\|_{H^{1}(\Omega)}+\left\|p-\bar{p}_{h}\right\|_{L^{2}(\Omega)} \leq c h^{\frac{3}{2}} .
$$

Proof. Clearly $F_{h} \in C^{1}\left(B_{1}(u) ; H^{1}\left(\Omega ; \mathbb{R}^{d}\right)\right)$ and $D F_{h}(u)=I-S_{h}$ with $S_{h} \in L\left(H^{1}\left(\Omega ; \mathbb{R}^{d}\right)\right)$. From the uniform ellipticity of $a_{h}$ (which follows from Lemma 3.2 and Lemma 3.3), the uniform continuity of $N_{h}$ on $H^{1}\left(\Omega ; \mathbb{R}^{d}\right)^{3}$ and (4.3) we conclude that $\left\|S_{h}\right\|_{L\left(H^{1}(\Omega)\right)} \leq 1 / 2$ provided $\operatorname{Re}\|f\|_{L^{2}(\Omega)}$ is small enough. Therefore, $D F_{h}(u)$ is an isomorphism of $H^{1}\left(\Omega ; \mathbb{R}^{d}\right)$ and

$$
\left\|D F_{h}(u)^{-1}\right\|_{L\left(H^{1}(\Omega)\right)} \leq \gamma \quad \text { uniformly in } h .
$$

Next, we want to estimate $\epsilon_{h}:=\left\|F_{h}(u)\right\|_{H^{1}}$. According to the definition of $F_{h}$ we may write $F_{h}(u)=u-\bar{w}_{h}$ where $w_{h} \in X_{h}$ is the solution of the following problem:

$$
\begin{aligned}
a_{h}\left(w_{h}, v_{h}\right)+b_{h}\left(v_{h}, p_{h}\right) & =\int_{\tilde{\Omega}_{h}} f \circ G_{h} \cdot v_{h}-N_{h}\left(u \circ G_{h}, u \circ G_{h}, v_{h}\right) & & \text { for all } v_{h} \in X_{h} \\
b_{h}\left(w_{h}, q_{h}\right) & =0 & & \text { for all } q_{h} \in M_{h} .
\end{aligned}
$$

Since $(u, p)$ is a solution of $(1.1,1.3,1.4)$ we also have

$$
\begin{aligned}
a(u, v)+b(v, p) & =\int_{\Omega} f \cdot v-\int_{\Omega}(u \cdot \nabla) u \cdot v & & \text { for all } v \in X \\
b(w, q) & =0 & & \text { for all } q \in M .
\end{aligned}
$$

Just as in the proof for the linear case we may now deduce an error relation for $u-\bar{w}_{h}$ :

$$
\begin{aligned}
a\left(u-\bar{w}_{h}, \bar{v}_{h}\right)+b\left(\bar{v}_{h}, p-\bar{p}_{h}\right)= & a_{h}\left(w_{h}, v_{h}\right)-a\left(\bar{w}_{h}, \bar{v}_{h}\right)+b_{h}\left(v_{h}, p_{h}\right)-b\left(\bar{v}_{h}, \bar{p}_{h}\right) \\
& +\int_{\Omega} f \cdot \bar{v}_{h}-\int_{\tilde{\Omega}_{h}} f \circ G_{h} \cdot v_{h}+\int_{\partial \Omega}(n \sigma(u, p) n) \bar{v}_{h} \cdot n \\
& -\int_{\Omega}(u \cdot \nabla) u \cdot \bar{v}_{h}+N_{h}\left(u \circ G_{h}, u \circ G_{h}, v_{h}\right) \\
b\left(u-\bar{w}_{h}, \bar{q}_{h}\right)= & b_{h}\left(w_{h}, q_{h}\right)-b\left(\bar{w}_{h}, \bar{q}_{h}\right)
\end{aligned}
$$

for all $v_{h} \in X_{h}, q_{h} \in M_{h}$.

From Lemma 4.3 below for the case $w=u \circ G_{h}$ we infer

$$
\sup _{v_{h} \in X_{h} \backslash\{0\}} \frac{\left|\int_{\Omega}(u \cdot \nabla) u \cdot \bar{v}_{h}-N_{h}\left(u \circ G_{h}, u \circ G_{h}, v_{h}\right)\right|}{\left\|\bar{v}_{h}\right\|_{H^{1}(\Omega)}} \leq c h^{\frac{3}{2}} .
$$


Combining this estimate with the argument in Theorem 3.4 we arrive at

$$
\epsilon_{h}=\left\|F_{h}(u)\right\|_{H^{1}(\Omega)}=\left\|u-\bar{w}_{h}\right\|_{H^{1}(\Omega)} \leq c h^{\frac{3}{2}}
$$

which together with (4.5) implies

$$
\left\|D F_{h}(u)^{-1} F_{h}(u)\right\|_{H^{1}(\Omega)} \leq \gamma\left\|F_{h}(u)\right\|_{H^{1}(\Omega)} \leq c h^{\frac{3}{2}} .
$$

Furthermore, it is not hard to prove that

$$
\left\|D F_{h}(v)-D F_{h}(w)\right\|_{L\left(H^{1}(\Omega)\right)} \leq K\|v-w\|_{H^{1}(\Omega)} \quad \text { for all } v, w \in B_{1}(u) .
$$

From $(4.5,4.7,4.8)$ we see that $(4.4)$ is satisfied provided $0<h \leq h_{5}$ and Theorem 4.1 implies that the equation $F_{h}(v)=0$ has a unique solution $\bar{u}_{h} \in B_{c h} \frac{3}{2}(u)$ or in other words

$$
\left\|u-\bar{u}_{h}\right\|_{H^{1}(\Omega)} \leq c h^{\frac{3}{2}}
$$

Furthermore, $u_{h}=\bar{u}_{h} \circ G_{h}$ solves (4.1).

Before we give a corresponding bound for the pressure we prove a lemma which deals with the nonlinearity and which has already been used above.

Lemma 4.3. Let $(u, p) \in H^{3}\left(\Omega, \mathbb{R}^{d}\right) \times H^{2}(\Omega)$ be the solution of $(1.1,1.3,1.4)$ and $w \in H^{1}\left(\tilde{\Omega}_{h}, \mathbb{R}^{d}\right)$. Then

$$
\sup _{v_{h} \in X_{h} \backslash\{0\}} \frac{\left|\int_{\Omega}(u \cdot \nabla) u \cdot \bar{v}_{h}-N_{h}\left(w, w, v_{h}\right)\right|}{\left\|\bar{v}_{h}\right\|_{H^{1}}} \leq c\left\|u-w \circ G_{h}^{-1}\right\|_{H^{1}}\left(\|u\|_{H^{1}}+\left\|w \circ G_{h}^{-1}\right\|_{H^{1}}\right)+\operatorname{ch}^{\frac{3}{2}} .
$$

Proof. Using integration by parts together with (1.3) we get

$$
\int_{\Omega}(u \cdot \nabla) u \cdot \bar{v}_{h}=\frac{1}{2} \int_{\Omega}(u \cdot \nabla) u \cdot \bar{v}_{h}-\frac{1}{2} \int_{\Omega}(u \cdot \nabla) \bar{v}_{h} \cdot u
$$

so that

$$
\begin{aligned}
\int_{\Omega}(u \cdot \nabla) u \cdot \bar{v}_{h}-N_{h}\left(w, w, v_{h}\right)= & \frac{1}{2}\left(\int_{\Omega}(u \cdot \nabla) u \cdot \bar{v}_{h}-\int_{\tilde{\Omega}_{h}}(w \cdot \nabla) w \cdot v_{h}\right) \\
& -\frac{1}{2}\left(\int_{\Omega}(u \cdot \nabla) \bar{v}_{h} \cdot u-\int_{\tilde{\Omega}_{h}}(w \cdot \nabla) v_{h} \cdot w\right) .
\end{aligned}
$$

The transformation rule and (3.2) imply

$$
\begin{aligned}
\left|\int_{\Omega}(u \cdot \nabla) u \cdot \bar{v}_{h}-\int_{\tilde{\Omega}_{h}}(w \cdot \nabla) w \cdot v_{h}\right|= & \left|\int_{\tilde{\Omega}_{h}}\left(u \circ G_{h} \cdot \nabla\right) u \circ G_{h} \cdot v_{h}\right| \operatorname{det} G_{h}\left|-\int_{\tilde{\Omega}_{h}}(w \cdot \nabla) w \cdot v_{h}\right| \\
\leq & \left|\int_{\tilde{\Omega}_{h}}\left(\left(u \circ G_{h} \cdot \nabla\right) u \circ G_{h} \cdot v_{h}-(w \cdot \nabla) w \cdot v_{h}\right)\right| \\
& +c \sum_{T \cap \partial \Omega \neq \emptyset}\|u\|_{C^{1}}^{2} h(\tilde{T}) \int_{\tilde{T}}\left|v_{h}\right| \\
\leq & c\left\|u \circ G_{h}-w\right\|_{H^{1}}\left(\left\|u \circ G_{h}\right\|_{H^{1}}+\|w\|_{H^{1}}\right)\left\|v_{h}\right\|_{H^{1}} \\
& +c \sum_{T \cap \partial \Omega \neq \emptyset} h(\tilde{T})^{\frac{d}{2}+1}\left\|v_{h}\right\|_{L^{2}(\tilde{T})} \\
\leq & c\left\|u-w \circ G_{h}^{-1}\right\| H_{H^{1}}\left(\|u\|_{H^{1}}+\left\|w \circ G_{h}^{-1}\right\|_{H^{1}}\right)\left\|\bar{v}_{h}\right\|_{H^{1}}+c h^{\frac{3}{2}}\left\|\bar{v}_{h}\right\|_{H^{1}} .
\end{aligned}
$$


Here we also employed the continuity of the trilinear form $(u, v, w) \mapsto \int_{\tilde{\Omega}_{h}}(u \cdot \nabla) v \cdot w$ on $\left(H^{1}\left(\tilde{\Omega}_{h}, \mathbb{R}^{d}\right)\right)^{3}$. The second term can be treated in the same way and the lemma follows.

In order to obtain the pressure bound we now write down an error relation analogous to (4.6) with $u-\bar{w}_{h}$ replaced by $u-\bar{u}_{h}$ and $N_{h}\left(u \circ G_{h}, u \circ G_{h}, v_{h}\right)$ replaced by $N_{h}\left(u_{h}, u_{h}, v_{h}\right)$. Observing that $\left\|u_{h}\right\|_{H^{1}} \leq c \operatorname{Re}\|f\|_{L^{2}}$ Lemma 4.3 implies

$$
\sup _{v_{h} \in X_{h} \backslash\{0\}} \frac{\left|\int_{\Omega}(u \cdot \nabla) u \cdot \bar{v}_{h}-N_{h}\left(u_{h}, u_{h}, v_{h}\right)\right|}{\left\|\bar{v}_{h}\right\|_{H^{1}(\Omega)}} \leq c\left\|u-\bar{u}_{h}\right\|_{H^{1}(\Omega)}+c h^{\frac{3}{2}}
$$

so that the argument in Theorem 3.4 gives

$$
\left\|p-\bar{p}_{h}\right\|_{L^{2}(\Omega)} \leq c h^{\frac{3}{2}}
$$

This concludes the proof of Theorem 4.2 .

\section{Numerical RESUlts}

From a numerical point of view it is quite simple to treat the boundary condition incorporated in $X_{h}$, see also [2]. If one uses iterative methods to solve problem (2.7) or (4.1) (for example iterative solvers based on the Schur complement, see e.g. $[2,6,19])$, then the core method consists of solving the elliptic problem

$$
a_{h}\left(u_{h}, v_{h}\right)=\left\langle\chi, v_{h}\right\rangle \quad \text { for all } v_{h} \in X_{h}
$$

with some right hand side $\chi$. Now consider

$$
\tilde{X}_{h}:=\left\{v_{h} \in C^{0}\left(\bar{\Omega}_{h} ; \mathbb{R}^{d}\right) \mid v_{h \mid \tilde{T}} \in \mathcal{P}_{2}(\tilde{T})^{d}\right\}
$$

the space of all piecewise quadratics (without enforced boundary condition). With $v_{h} \in \tilde{X}_{h}$ we associate its representation by a vector of nodal values $\underline{v}=\left\{v_{p}\right\}_{p}=\left\{v_{p}^{1}, v_{p}^{2}, v_{p}^{3}\right\}_{p} \subseteq \underline{\tilde{X}}_{h}$ such that

$$
v_{h}=\sum_{p \in \mathcal{N}_{h}} v_{p} \varphi_{p}=\sum_{p \in \mathcal{N}_{h}}\left(v_{p}^{1}, v_{p}^{2}, v_{p}^{3}\right)^{T} \varphi_{p}
$$

with $\left\{\varphi_{p}\right\}_{p}$ the nodal basis of $\tilde{X}_{h}$, i.e.

$$
\varphi_{p}(q)=\delta_{p q} \quad \text { for all } q \in \mathcal{N}_{h}
$$

Accordingly define

$$
\underline{X}_{h}:=\left\{\underline{v} \in \underline{\tilde{X}}_{h} \mid v_{p} \cdot n_{p}=0 \forall p \in \mathcal{N}_{h} \cap \partial \tilde{\Omega}_{h}\right\}
$$

where $n_{p}=n\left(G_{h}(p)\right)$. Define the projection $P: \underline{\tilde{X}}_{h} \rightarrow \underline{X}_{h}$ by

$$
(P \underline{v})_{p}= \begin{cases}v_{p}-v_{p} \cdot n_{p} n_{p} & \text { if } p \in \mathcal{N}_{h} \cap \partial \tilde{\Omega}_{h} \\ v_{p} & \text { else }\end{cases}
$$

and the operator $A_{h}: \underline{\tilde{X}}_{h} \rightarrow \underline{\tilde{X}}_{h}$ by

$$
a_{h}\left(v_{h}, w_{h}\right)=\left(A_{h} \underline{v}, \underline{w}\right)_{\mathbb{R}^{3 N}} \quad \text { for all } v_{h}, w_{h} \in \tilde{X}_{h} .
$$


TABLE 1. Numerical results for $5 \times 3$ bisection steps.

\begin{tabular}{|r|r|r|l|l|l|r|}
\hline level & $\sharp$ tetrahedra & $\sharp$ nodes & $\left\|u_{h}-u\right\|_{H^{1}}$ & $\mathrm{EOC}$ & $\left\|p_{h}-p\right\|_{L^{2}}$ & $\mathrm{EOC}$ \\
\hline 0 & 6 & 27 & $0.6985 \mathrm{E}+01$ & - & $0.1976 \mathrm{E}+02$ & - \\
\hline 3 & 48 & 125 & $0.4607 \mathrm{E}+01$ & 0.60 & $0.8430 \mathrm{E}+01$ & 1.23 \\
\hline 6 & 384 & 729 & $0.1566 \mathrm{E}+01$ & 1.56 & $0.2543 \mathrm{E}+01$ & 1.73 \\
\hline 9 & 3072 & 4913 & $0.4530 \mathrm{E}+00$ & 1.79 & $0.6862 \mathrm{E}+00$ & 1.89 \\
\hline 12 & 24576 & 35937 & $0.1224 \mathrm{E}+00$ & 1.89 & $0.1791 \mathrm{E}+00$ & 1.94 \\
\hline 15 & 196608 & 274625 & $0.3301 \mathrm{E}-01$ & 1.89 & $0.4599 \mathrm{E}-01$ & 1.96 \\
\hline
\end{tabular}

Here, $(\cdot, \cdot)_{\mathbb{R}^{3 N}}$ denotes the Eucledian inner product in $\mathbb{R}^{3 N}, N=\operatorname{dim} \tilde{X}_{h}$. Then (5.1) is equivalent to: Find $\underline{u}=P \underline{\tilde{u}}, \underline{\tilde{u}} \in \underline{\tilde{X}}_{h}$ such that

$$
\left(A_{h} P \underline{\tilde{u}}, P \underline{v}\right)_{\mathbb{R}^{3 N}}=\left(P^{T} A_{h} P \underline{\tilde{u}}, \underline{v}\right)_{\mathbb{R}^{3 N}}=\left(P A_{h} P \underline{\tilde{u}}, \underline{v}\right)_{\mathbb{R}^{3 N}}=(P \underline{\chi}, \underline{v})_{\mathbb{R}^{3 N}}
$$

for all $\underline{v} \in \underline{\tilde{X}}_{h}$ and with $\chi_{p}:=\left\langle\chi, \varphi_{p}\right\rangle$. Using an iterative procedure to solve (5.1) requires the evaluation of $a_{h}\left(v_{h}, w_{h}\right)$ which by $(5.2)$ is equivalent to working on $\tilde{X}_{h}$ and projecting after each matrix times vector operation. Note that the projection $P$ is a simple and numerically cheap operation. A similar consideration also holds in the case when the matrix corresponding to $A_{h}$ is preconditioned by e.g. a multilevel procedure.

Since it is difficult to find a nontrivial explicit solution to $(1.1,1.3-1.4)$ we consider an example which is not entirely covered by our error analysis but in which the setting is even more complicated. Let $\Omega:=B_{1}(0) \subseteq \mathbb{R}^{3}$ and choose the exterior force $f$ in such a way that the pair $(u, p)$ with

$$
\begin{aligned}
& u(x, y, z)=\left[\begin{array}{c}
2 x z\left(x^{2}+y^{2}\right) \\
2 y z\left(x^{2}+y^{2}\right) \\
4\left(x^{2}+y^{2}\right)\left(1-\frac{3}{2}\left(x^{2}+y^{2}\right)-z^{2}\right)
\end{array}\right], \\
& p(x, y, z)=16\left(z-\frac{z^{3}}{3}\right)
\end{aligned}
$$

solves (1.1) and (1.3). Instead of (1.4) we have nontrivial tangential stresses. The above solution is similar to Hill's spherical vortex, see for instance [3].

Note also that in view of the symmetry of $\Omega u$ is only unique up to a rigid body rotation. We discretize $\Omega$ by choosing a macro-triangulation and then refine this coarse grid by the bisection method introduced in [1]. The normal $n\left(G_{h}(p)\right)$ is given by $n\left(G_{h}(p)\right)=p /|p|$. Table 1 shows the resulting errors and experimental orders of convergence (EOC) for successive refinements of the macro triangulation. "Level" denotes the number of refinement steps. Note that 3 refinement steps of the bisection method yield a triangulation with halved grid size.

Figure 2 shows the solution for refinement level $4 \times 3$. 


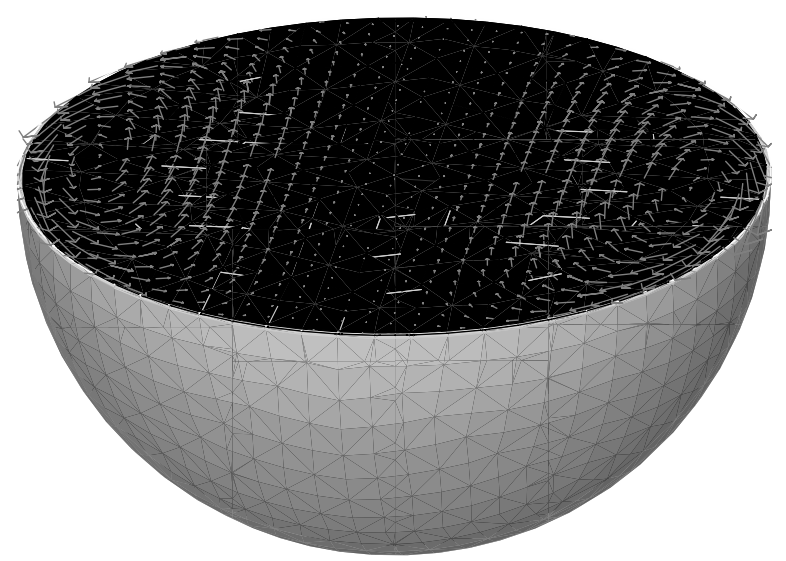

Figure 2. Solution on a clipping plane, refinement level $4 \times 3$.

The authors want to thank B. Höhn for performing the numerical example.

\section{REFERENCES}

[1] E. Bänsch, Local Mesh Refinement in 2 and 3 Dimensions. Impact Comp. Sci. Eng. 3 (1991) 181-191.

[2] E. Bänsch and B. Höhn, Numerical treatment of the Navier-Stokes equations with slip-boundary conditions. Preprint 9/1998, Universität Freiburg; SIAM J. Sci. Comp. (submitted).

[3] G.K. Batchelor, An Introduction to Fluid Dynamics. University Press, Cambridge (1970).

[4] C. Bernardi, Optimal finite element interpolation on curved domains. SIAM J. Numer. Anal. 26 (1989) 212-234.

[5] F. Brezzi and M. Fortin, Mixed and hybrid finite element methods. Springer, New York (1991).

[6] M.O. Bristeau, R. Glowinski and J. Periaux, Numerical methods for the Navier-Stokes equations. Application to the simulation of compressible and incompressible flows. Comput. Phys. Rep. 6 (1987) 73-188.

[7] P.G. Ciarlet, The finite element method for elliptic problems. North-Holland, Amsterdam (1977).

[8] K. Deimling, Nonlinear Functional Analysis. Springer, Berlin (1985).

[9] V. Girault and P.A. Raviart, Finite element methods for Navier-Stokes equations, Theory and Algorithms. Springer, Berlin (1986).

[10] C. Cuvelier and J.M. Driessen, Thermocapillary free boundaries in crystal growth. J. Fluid Mech. 169 (1986) 1-26.

[11] S.F. Kistler and L.E. Scriven, Coating flow theory by finite element and asymptotic analysis of the Navier-Stokes system. Internat. J. Numer. Methods Fluids 4 (1984) 207-229.

[12] P. Knobloch, Variational Crimes in a Finite Element Discretization of 3D Stokes Equations with Nonstandard Boundary Conditions. Preprint MBI-97-4, University of Magdeburg (1997).

[13] M. Lenoir, Optimal isoparametric finite elements and error estimates for domains involving curved boundaries. SIAM J. Numer. Anal. 23 (1986) 562-580.

[14] J.A. Nitsche, On Korn's second inequality. RAIRO Anal. Numér. 15 (1981) 237-248.

[15] F. Otto, Die Babuška-Brezzi Bedingung für das Taylor-Hood-Element. Diploma thesis, University of Bonn, Germany (1990).

[16] H. Saito and L.E Scriven, Study of Coating Flow by the Finite Element Method. J. Comput. Phys. 42 (1981) 53-76.

[17] D. Schwabe, Surface-Tension-Driven Flow in Crystal Growth Melts, in Crystal Growth, Properties and Applications 11. Springer, Berlin (1988).

[18] S.A. Solonnikov and V.E. Ščadilov, On a boundary value problem for a stationary system of Navier-Stokes equations. Proc. Steklov Inst. Math. 125 (1973) 186-199.

[19] R. Verfürth, A combined conjugate gradient-multigrid algorithm for the numerical solution of the Stokes problem. IMA J. Numer. Anal. 4 (1984) 441-455.

[20] R. Verfürth, Finite element approximation of steady Navier-Stokes equations with mixed boundary conditions. Math. Modelling Numer. Anal. 19 (1985) 461-475.

[21] R. Verfürth, Finite element approximation of incompressible Navier-Stokes equations with slip boundary condition. Numer. Math. 50 (1987) 697-721.

[22] R. Verfürth, Finite element approximation of incompressible Navier-Stokes equations with slip boundary condition II. Numer. Math. 59 (1991) 615-636. 